\title{
Characterization of Regulatory and Transporter Genes in the Biosynthesis of Anti-Tuberculosis Ilamycins and Production in a Heterologous Host
}

\author{
Jianqiao He ${ }^{1,2}$, Xin Wei ${ }^{1,2}$, Zhijie Yang ${ }^{1,2}$, Yan $\mathrm{Li}^{1}$, Jianhua $\mathrm{Ju}^{1,2,3}$ and Junying Ma ${ }^{1,3, *(\mathbb{D})}$ \\ 1 CAS Key Laboratory of Tropical Marine Bio-Resources and Ecology, Guangdong Key Laboratory of Marine \\ Materia Medica, RNAM Center for Marine Microbiology, South China Sea Institute of Oceanology, Chinese \\ Academy of Sciences, Guangzhou 510301, China; 13409727100@163.com (J.H.); weixin1011@126.com (X.W.); \\ yzjie6@126.com (Z.Y.); ly505479742@126.com (Y.L.); jju@scsio.ac.cn (J.J.) \\ 2 University of Chinese Academy of Sciences, Beijing 100049, China \\ 3 Key Special Project for Introduced Talents Team of Southern Marine Science and Engineering Guangdong \\ Laboratory, Guangzhou 510301, China \\ * Correspondence: majunying@scsio.ac.cn; Tel.: +(86)20-34066449
}

Received: 20 March 2020; Accepted: 14 April 2020; Published: 17 April 2020

\begin{abstract}
Ilamycins are cyclopeptides with novel structures that have been isolated from different Actinomycetes. They showed strong anti-tuberculosis activity and could serve as important anti-tuberculosis drug leads. The functions of the pre-tailoring and the post-tailoring genes in the biosynthesis of ilamycins have been elucidated, but the functions of the regulatory and transporter genes remain elusive. We reported herein the functions of four genes in ilamycin biosynthetic gene cluster (ila BGC) including two regulatory genes (ila $A$ and ilaB) and two transporter genes (ilaJ and ilaK) and the heterologous expression of ila BGC. The IlaA and IlaB were unambiguously shown to be negative and positive regulator of ilamycins biosynthesis, respectively. Consistent with these roles, inactivation of ila $A$ and ilaB (independent of each other) was shown to enhance and abolish the production of ilamycins, respectively. Total yields of ilamycins were enhanced 3.0-fold and 1.9-fold by inactivation of $i l a A$ and overexpression of ilaB compared to those of in the Streptomyces atratus SCSIO ZH16, respectively. In addition, the ila BGC was successfully expressed in Streptomyces coelicolor M1152, which indicated that all biosynthetic elements for the construction of ilamycins were included in the PAC7A6. These results not only lay a foundation for further exploration of ilamycins, but also provide the genetic elements for synthetic biology.
\end{abstract}

Keywords: marine-derived Streptomyces; ilamycins; regulator; ABC-transporter; heterologous expression

\section{Introduction}

Marine derived Streptomyces have been identified as sources for novel natural products that are of great interest to drug discovery and development [1-3]. Non-ribosomal peptides are an important class of natural products known for their structural diversity and bioactivity; many antitumor and anti-infective drugs are non-ribosomal peptides [4]. For example, romidepsin, used to treat T-cell lymphoma [5-7], daptomycin and vancomycin, used to treat multidrug-resistant infections [8-12], and capreomycin, used for tuberculosis (TB) [13], are all non-ribosomal peptides. Ilamycins, also known as rufomycins, are potent anti-TB cyclic heptapeptides containing three rare building blocks, L-3-nitrotyrosine, L-2-amino-4-hexenoic acid, and L-tryptophan, in which the indole nitrogen is alkylated with an isoprene or a modified isoprene unit. Ilamycins were cyclopeptides isolated from several strains [14-16]. Recently, eight more ilamycin analogs, ilamycins $B_{1}(\mathbf{1}), B_{2}(2)$, $C_{1}(3), C_{2}(4), D(5), E_{1}(6), E_{2}$, and $F$ (Figure $1 A$ ), were isolated by our group from a marine 
Streptomyces atratus SCSIO ZH16 and its engineered strain [17]. Bioactivity studies revealed that ilamycins, except ilamycin $\mathrm{B}_{1}$, have potent anti-TB activities $[17,18]$, and that ilamycin $\mathrm{E}$ has the strongest anti-TB activity at a minimal inhibition concentration of $9.8 \mathrm{nM}$ [17]. Anti-TB mechanistic studies revealed that these ilamycins are bactericidal to Mycobacterium tuberculosis through their inhibition of caseinolytic protease complex component ( $\mathrm{ClpC} 1$ ) and subsequent modulation of the degradation of intracellular proteins [19]. Ilamycin $C$ and ilamycin $E$ have selective anti-triple-negative breast cancer activity and have been found to act on the interleukin-6/signal transducer and activator of transcription 3 (IL-6/STAT3) and endoplasmic reticulum stress-CCAAT-enhancer-binding protein homologous protein-B-cell lymphoma 2 (ER stress-CHOP-Bcl2) signaling pathways, respectively $[20,21]$. The biosynthesis of the rare L-3-nitrotyrosine and L-2-amino-4-hexenoic acid units as well as two post-tailoring steps in the ilamycin biosynthetic pathway have been studied by our group and others through a series of gene inactivation, precursor chemical complementation, isotope-labeled precursor feeding experiments as well as structural elucidation of three intermediates from the respective mutants $[17,22]$. However, the functions of the regulatory and transporter genes in the biosynthesis of these compounds in these Streptomyces strains have not been characterized.

A)

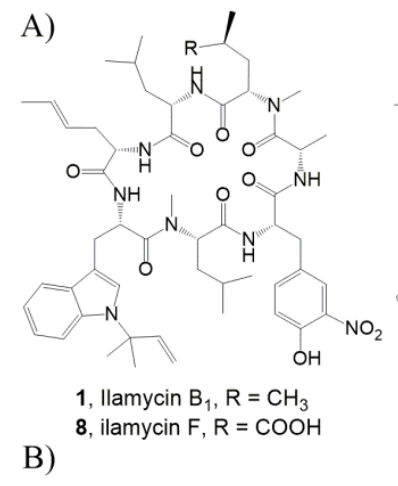

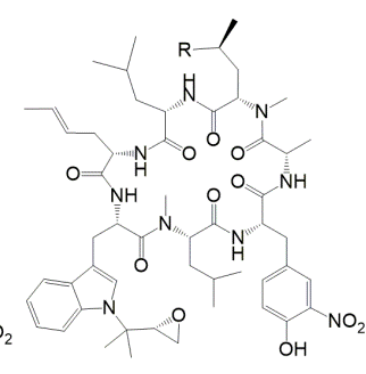

2, llamycin $\mathrm{B}_{2}, \mathrm{R}=\mathrm{CH}_{3}$ 5, llamycin $\mathrm{D}, \mathrm{R}=\mathrm{COOH}$

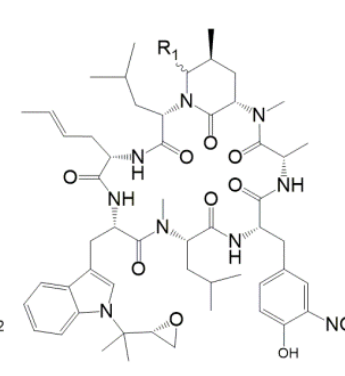

3, Ilamycin $\mathrm{C}_{1}, \mathrm{R}_{1}=\alpha \mathrm{OH}$ 4, llamycin $\mathrm{C}_{2}, \mathrm{R}_{1}=\beta \mathrm{OH}$

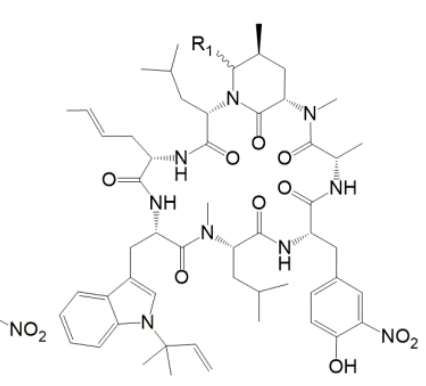

6, llamycin $\mathrm{E}_{1}, \mathrm{R}_{1}=\alpha \mathrm{OH}$

7, llamycin $E_{2}, R_{1}=\beta O H$

$(-3) \operatorname{orf}(-1)$

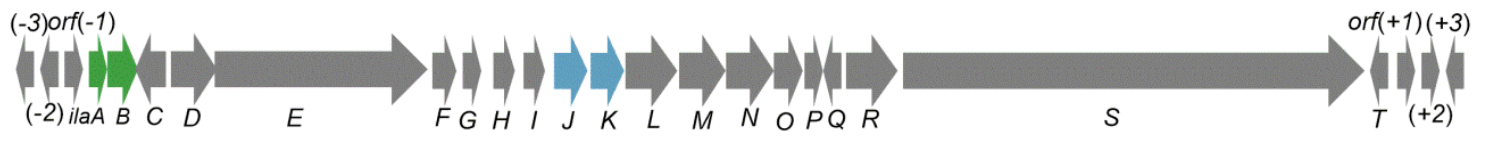

Figure 1. The structures and biosynthetic gene cluster of ilamycins. (A) The structures of ilamycins. (B) The genetic organization of ilamycin biosynthetic gene cluster, the genes marked with green and pale blue color encode the regulatory and transporter genes, respectively.

Herein, we aimed to identify and characterize the functions of the regulators and transporters involved in the biosynthesis of ilamycins, further use them to construct high-yield strains, and express the biosynthetic gene cluster of ilamycins in Streptomyces coelicolor M1152.

\section{Results}

\subsection{Identification of IlaA as a LysR Family Regulator and IlaB as a Streptomycin Biosynthesis Operon} Regulator by Bioinformatics Analysis

The biosynthetic gene cluster of ilamycins consisted of 20 genes (Figure 1B), which had been identified in our previous study [17]. IlaA and IlaB were resided in the upstream of ilamycin biosynthetic gene cluster (ila). IlaA was firstly annotated as a LysR family transcriptional regulator. However, our analysis using the basic local alignment search tool (BLAST) revealed that it was annotated as a hypothetical protein, and only one homologue sequence derived from Liparis tanakae was found, which means its function remains elusive.

IlaB was annotated as a streptomycin biosynthesis operon regulator by BLAST analysis. To understand the sequence characteristics of IlaB, a sequence alignment of IlaB and four other homologs, DtpR2 [23], StaQ [24], StrR [25], and Veg1 [26], was performed with Clustal Omega [27], 
and the alignment result was viewed in ESPript [28], as shown in Figure 2. The online Pfam database was used to analyze the domains of streptomycin biosynthesis operon regulator [29]. The characteristics of streptomycin biosynthesis operon regulator are as follows: (i) it contains a ParB-like nuclease domain in the N-terminal of IlaB (49-125 amino acids for IlaB), which is involved in many cellular processes, such as cell cycle control, cell division, sporulation, and chromosome partitioning; (ii) there is a homeodomain-like domain in the middle of IlaB (137-173 amino acids for IlaB), which possesses multiple regulatory functions such as stress response, metabolism, transport/transfer, or regulation; (iii) a winged helix-turn-helix domain similar to that seen in AsnC/LacI family regulators resides almost the middle of IlaB (205-238 amino acids for IlaB).
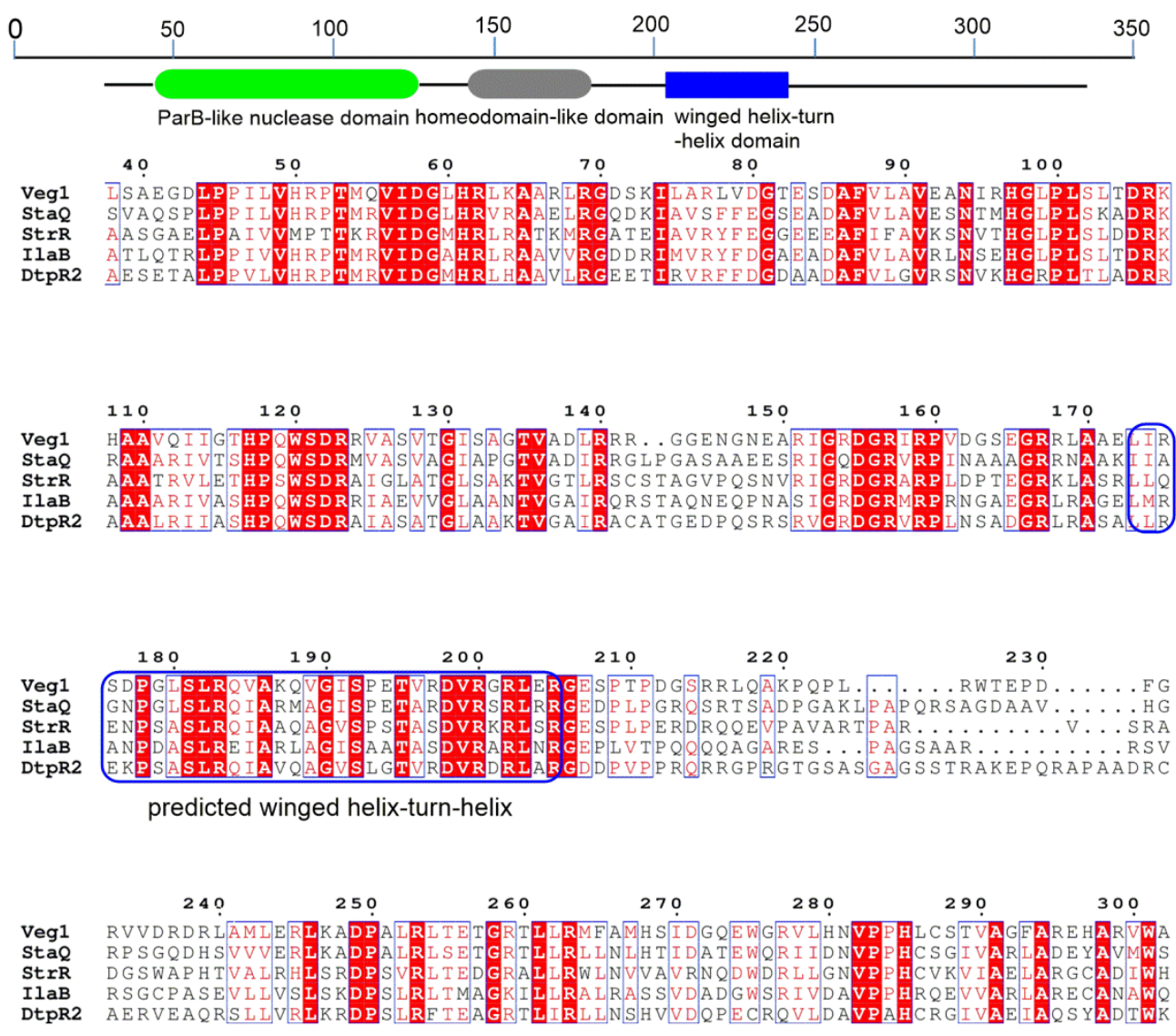

Figure 2. Domains in IlaB and primary sequence alignment of IlaB (ASX95224) with its homologues including StaQ (GenBank accession no. AAM80553) from Streptomyces toyocaensis NRRL 15009, StrR (GenBank accession no. BAG22760) from Streptomyces griseus subsp. griseus NBRC 13350, Veg1 (GenBank accession no. ACJ60943) from uncultured bacterium, DtpR2 (GenBank accession no. AJI44174) from Saccharothix algeriensis. The sequence encoding a predicted winged helix-turn-helix DNA binding motif is noted with a blue box.

\subsection{Identification of IlaA and IlaB as Negative and Positive Regulators Regulating the Production of Ilamycins}

To investigate the functions of IlaA and IlaB in the biosynthesis of ilamycins, $\triangle$ ila $A$ and $\triangle$ ilaB mutants were constructed by the methods described previously [17]. The phenotype and the genotype were confirmed by (apramycin-resistance/kanamycin-sensitivity) $\mathrm{Apr}^{\mathrm{R}} / \mathrm{Kan}^{\mathrm{S}}$ and polymerase chain reaction (PCR) analyses. The PCR results are shown in Figures S1 and S2. In order to characterize the production of ilamycins in these two $\triangle i l a A$ and $\triangle$ ilaB mutants, the mutants of $\triangle i l a A$ and $\triangle$ ilaB were fermented under the conditions described in our previous studies [17], with the wild-type as a control. High-performance liquid chromatography (HPLC) analysis of the fermentation media extracts revealed that $\triangle$ ilaA extracts produced higher titers of ilamycins (Figure 3, trace ii) than those of the wild 
type (Figure 3, trace i). The total yields of ilamycins produced by the $\triangle i l a A$ mutants were enhanced 3.0-fold (11.76 mg/mL) when compared to those in the wild type $(3.92 \mathrm{mg} / \mathrm{mL})$ (Figure 3B). However, $\triangle i l a B$ completely abolished the production of ilamycins (Figure 3, trace iii). These results indicate that IlaA and IlaB might work as a negative regulator and a positive regulator, respectively, in the biosynthesis of ilamycins.

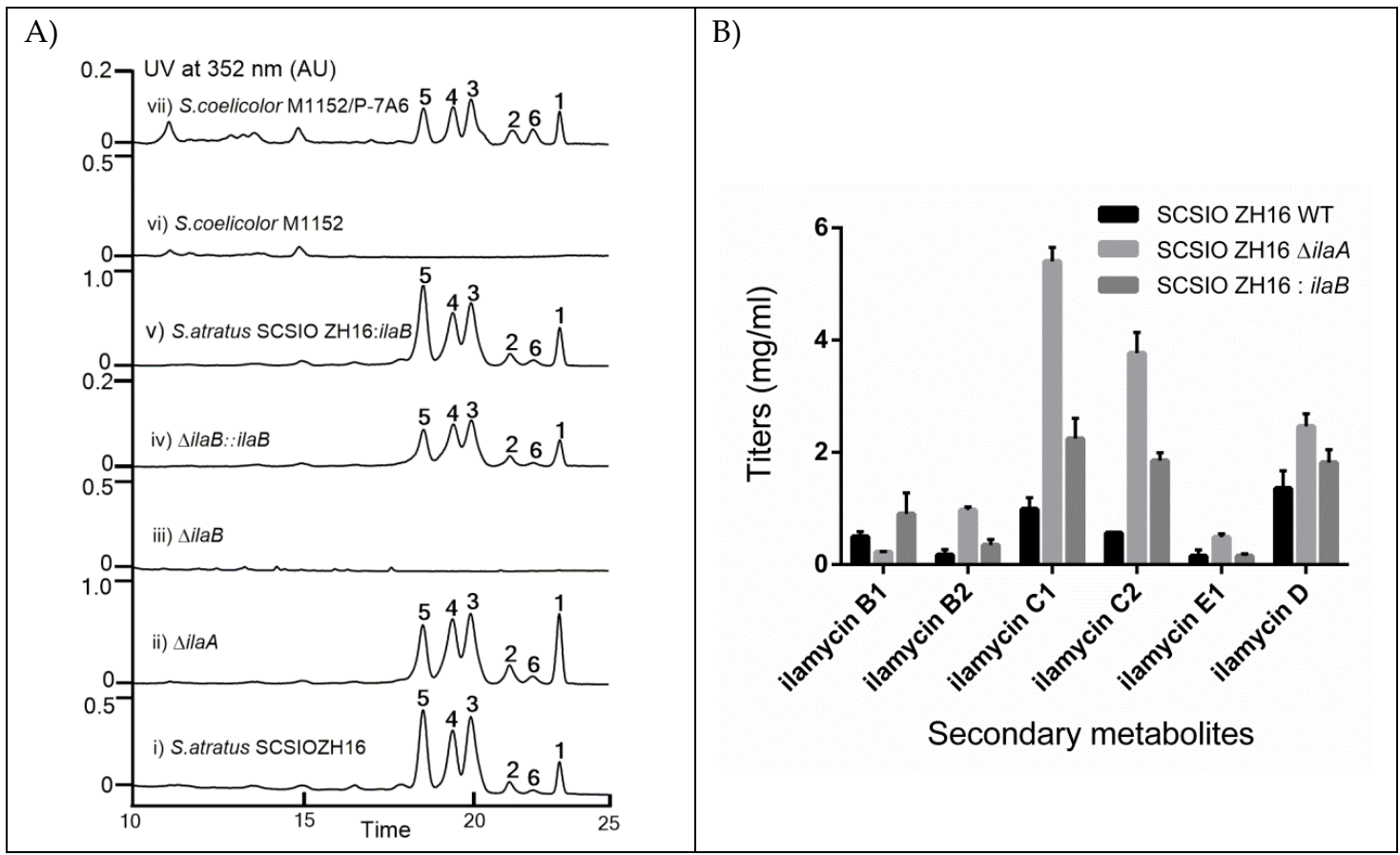

Figure 3. HPLC analysis of fermentation extracts and titers quantification. (A) HPLC analysis of fermentation of mutants and wild type. (i) S. atratus SCSIO ZH16, (ii) $\triangle$ ilaA, (iii) $\triangle$ ilaB, (iv) $\Delta$ ilaB::ilaB, (v) S. atratus SCSIO ZH16:ilaB, (vi) Streptomyces coelicolor M1152, (vii) S. coelicolor M1152-PAC7A6. (B) Comparative analysis of ilamycin yield between $\triangle i l a A$, overexpression IlaB mutant and the wild type strain. The values are mean of three different clones.

To verify whether the abrogation of ilamycin production in $\triangle i l a B$ mutant was indeed caused by the deletion of $i l a B$, a complementation experiment to $\triangle i l a B$ mutant was performed. The ila $B$ gene was expressed under the control of PermE* in pL646ATE vector in Escherichia coli ET12567/pUZ8002, which was introduced into $\triangle$ ilaB mutant by inter-genetic conjugation. The complement mutants $\triangle$ ilaB::ilaB-pL646ATE were fermented under standard conditions for ilamycin production. HPLC analysis showed that the production of ilamycins was restored in the complement mutant (Figure 3, trace iv) as those seen in the wild type. Taken together, these results conclusively establish the positive regulatory role of IlaB in the production of ilamycins.

\subsection{Overexpression of IlaB Enhanced the Production of Ilamycins}

Positive regulators play important roles in the biosynthesis of secondary metabolites, and their overexpression can directly improve the titers of these secondary metabolites. To examine whether $\mathrm{IlaB}$, as a positive regulator, could increase the yields of ilamycins, overexpression plasmids were constructed under the control of the PermE* in the pL646ATE vector and then introduced into S. atratus SCSIO ZH16 to generate ilaB overexpression mutants. With the wild type S. atratus SCSIO ZH16 as a control under the standard conditions for ilamycin production, overexpression mutants of $S$. atratus SCSIO ZH16:ilaB (Figure 3, trace v) were fermented. HPLC analysis of the fermentation media extracts revealed that the overall titer of ilamycins (1-6, Figure 1B) in S. atratus SCSIO ZH16:ilaB were enhanced 1.9 -fold $(7.45 \mathrm{mg} / \mathrm{mL}$ ) (Figure 3B) as compared to those of in the wild type. 


\subsection{Identification of IlaJ/K as Type I ABC-Exporter Transporters Mediating the Secretion of Ilamycins}

IlaJ and ilaK were found in the middle of the ilamycin gene cluster and annotated as typical adenosine triphosphate (ATP)-binding cassette (ABC) transporter and $\mathrm{ABC}-2$ type transporter, respectively, by BLAST analysis. The $\mathrm{ABC}$ transporter is a multi-domain protein consisting of four domains, two of which are transmembrane domains (TMD) and the other two are hydrophilic nucleotide-binding domains (NBD). The most common architecture of the ABC exporter protein is that of the so-called "half transporter," which contains single TMD and NBD domains and has to interact with another "half transporter" to gain function [30]. In order to investigate the sequence characteristics of IlaJ and IlaK, sequence alignment and transmembrane characteristics were analyzed with online software such as ESPript [28] and TMHMM server 2.0 (Http://www.cbs.dtu.dk/services/TMHMM/).

IlaJ/K showed high sequence similarity to other type I ABC-exporter transporters, such as DrrA/B [31], MtrA/B [32], TnrB2/B3 [33] and Atr29/30 [34], which involved in the secretion or self-defense for the producer of doxorubicin, mithramycin, tetronasin, and atratumycin, respectively. Conserved domains, such as Walker A (GXXGXGKS/T), Walker B $(\varphi \varphi \varphi \varphi \mathrm{DE}, \varphi$ represents hydroprobic amino acids), the $A B C$ signature (L/YSGGQM), the Q-loop, and the switch region were presented in IlaJ (Figure S3), which suggest that IlaJ could function as an ATP-binding subunit. The sequence alignment results of IlaK with their homologues revealed that the typical "EAAxxxGxxxxxxxxxIxLP" motif [35] of the importer was not found in IlaK and their homologues (Figure S4). Moreover, transmembrane analyses showed that IlaJ does not contain any transmembrane domain, whereas IlaK has six transmembrane domains similar with those in DrrB, MtrB, TnrB3, and Atr30. These results indicate that IlaJ and IlaK may work together, forming functional exporter units to mediate the secretion of ilamycins or the self-defense of S. atratus SCSIO ZH16 to ilamycins.

To investigate the roles of IlaJ and IlaK in the biosynthesis of anti-TB ilamycins, three mutants, $\Delta i l a J$, $\Delta i l a K$, and $\Delta i l a J K$, were constructed by the aforementioned method. Their phenotype and genotype were confirmed by $\mathrm{Apr}^{\mathrm{R}} / \mathrm{Kan}^{\mathrm{S}}$ and PCR analyses. PCR results are shown in Figures S5-S7. $\Delta$ ilaj, $\triangle$ ilaK, and $\Delta i l a j K$ were fermented under the same conditions with the wild type as a control. To characterize the possible functions of IlaJ and Ilak in secretion of ilamycins or self-defense of S. atratus SCSIO ZH16 to ilamycins, the titers of ilamycins in both of the wild type and three mutants were quantified by HPLC analysis (Figure 4A) and standard curve (Figure S8). The quantified results revealed that the overall titers of ilamycins in $\Delta i l a J, \Delta i l a K$, and $\Delta i l a J K$ were decreased to $69.5 \%(2.70 \mathrm{mg} / \mathrm{mL}), 59.9 \%$ $(2.34 \mathrm{mg} / \mathrm{mL})$, and $36.1 \%(1.42 \mathrm{mg} / \mathrm{mL})$ to those of in the wild types (Figure $4 \mathrm{~B})$. Especially, the titers of ilamycin $C_{1}$ and $C_{2}$ in those three mutants were decreased by $50 \%$ to $86 \%$ compared to those of in the wild type (Figure 4B). Based on these findings, we propose that IlaJ and IlaK play roles in the biosynthesis of ilamycins, such as the secretion of ilamycins or self-defense of S. atratus SCSIO ZH16 to ilamycins. 
A)

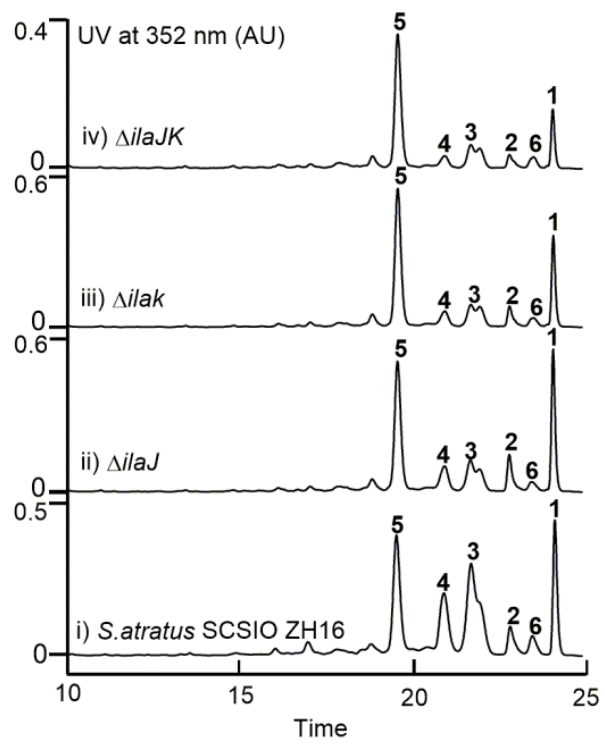

B)

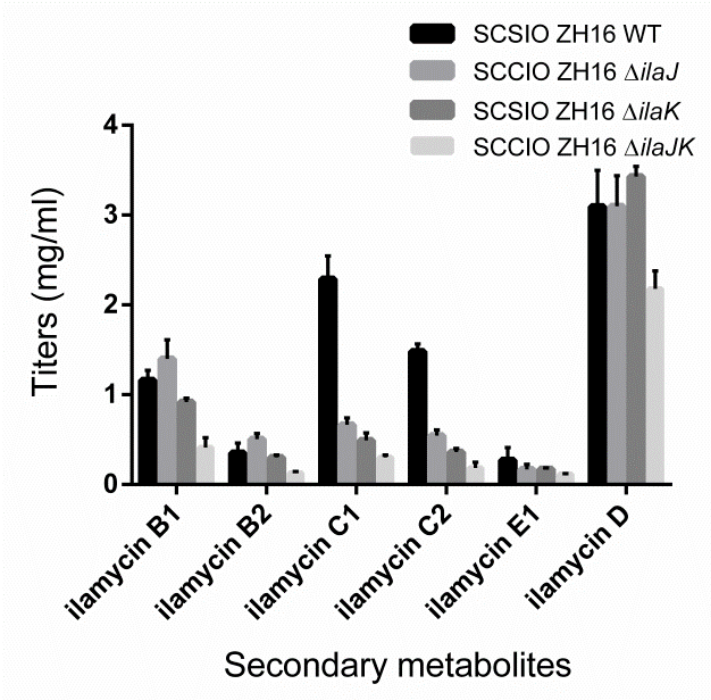

Figure 4. HPLC analysis of fermentation extracts and titers quantification. (A) (i) S. atratus SCSIO ZH16, (ii) $\Delta$ ilaJ, (iii) $\Delta$ ilak, (iv) $\Delta$ ilaJk. (B) Comparative analysis of ilamycin yield between $\Delta i l a J, \Delta i l a K$, and $\Delta i l a J K$ mutants and the wild type strain. The values are mean of two different clones.

\subsection{Heterologous Expression of the Ila Biosynthetic Gene Cluster (BGC) in Streptomyces Coelicolor M1152}

The biosynthetic gene cluster of ilamycins has been precisely characterized in our previous study [17]. To determine whether the ila BGC (ca $57 \mathrm{~kb})$ can be successfully expressed in a Streptomyces model, such as Streptomyces coelicolor M1152. A PAC7A6 containing the proposed whole ilamycin gene cluster was introduced into S. coelicolor M1152 by tri-parental intergeneric conjugation [34,36,37]. The correction the exconjugants was verified by PCR amplification of the genes in the ilamycin cluster (Figure S9). HPLC analyses of the fermentation products of the exconjugants revealed several new peaks (Figure 3, trace vii) when compared with the wild type S. coelicolor M1152 (Figure 3, trace vi) under the same conditions. To confirm whether these new peaks in the fermentation products of the exconjugants were ilamycins, the ultraviolet spectra and the retention times of the new peaks were further analyzed, and the results reveal that they were identical to those seen in the wild type S. atratus SCSIO ZH16 (Figure 3, trace i), but the overall titers of ilamycins produced in S. coelicolor M1152/PAC7A6 were just one quarter of those in the wild type S. atratus SCSIO ZH16. These results indicate that the biosynthetic elements for the construction of ilamycins are all included in the PAC7A6 and ilamycins can be produced in heterologous Streptomyces models, such as S. coelicolor M1152. Furthermore, this result provides a reference for the discovery of other new complex cyclic peptides by heterologous expression.

\section{Discussion}

Actinomycetes, especially the genus Streptomyces, are very important sources of bioactive natural products $[38,39]$. However, the production of bioactive compounds is strictly controlled by many factors, such as the nutritional status, $\mathrm{pH}$, temperature, dissolved oxygen, various environmental conditions, and regulators in their biosynthetic gene clusters [40,41]. Regulator genes and transport genes are important components of the secondary metabolite biosynthetic gene cluster, and they play important roles in changing the titers of these natural products.

IlaA is a small protein of unknown function. Our results revealed that IlaA functioned as a negative regulator in the biosynthesis of ilamycins. IlaB belongs to the streptomycin biosynthesis 
operon regulator family. The typical representative of this kind regulator is StrR, a pathway-specific activator in the biosynthesis of streptomycin in Streptomyces griseus and Streptomyces glaucescens. The molecular mechanisms studies of StrR revealed that it was a pathway specific transcriptional activator protein with multiple recognition sites in the biosynthesis of streptomycin and was depended on by nine transcriptional units within the streptomycin biosynthesis gene cluster [42,43]. In light of the high identity and the structure similarity between StrR and IlaB, we propose that IlaB may have a similar regulatory mechanism in the biosynthesis of ilamycins to that of StrR in the biosynthesis of streptomycin.

ATP-binding cassette (ABC) transporters belong to a large family of proteins that are widespread among living organisms. In bacteria, $\mathrm{ABC}$ transporters have a diverse range of functions, including an importer function mediating the uptake of nutrients and exporter function involving the secretion of various molecules, which are important for normal bacterial physiology [44,45]. The knockout of atr 29 and atr30 led to the complete abolishment of atratumycin in S. atratus SCSIO ZH16NS-80S both in the pellet and the supernatant media [34]. In this study, the knockout of ilaJ, ilaK, and ilaJK led to an obvious decrease in the total yields of ilamycins, especially for the decrease of ilamycin $C_{1}$ and $C_{2}$, which indicated that they played important roles in the secretion of ilamycins or self-defense to ilamycins in $S$. atratus SCSIO ZH16. It is noteworthy that with the surge in genome sequencing of Actinobacteria, more $\mathrm{ABC}$ transporters will be identified in the biosynthetic gene clusters of natural products, and their roles in secretion or self-defense will be elucidated in secondary metabolite-producing strains [46]. $\mathrm{ABC}$ transporters can be used to construct high-yield strains or antibiotic-resistant model strains for heterologous expression of other BGCs.

Heterologous expression not only plays important roles in the discovery of novel natural products in the genomic era, but also provides a way to verify the integrity of the BGCs or to characterize the function of biosynthetic pathways in a genetically amenable host. Due to the progress of large DNA fragment cloning technology, the success rates of heterologous expression of larger biosynthetic gene clusters have significantly increased, as seen in the cases of atratumycin [34], neoabyssomicins [37], A201A [47], and grincamycin [48]. The successful expression of ila BGC in a heterologous host, which not only suggests the possibility of heterologous expression in the discovery of new natural products, but also confirms the feasibility of verifying the integrity of big gene clusters.

\section{Conclusions}

In summary, the small protein (annotated as a LysR family regulator) encoded by ila $A$ and the streptomycin biosynthesis operon regulator encoded by ila $B$ were demonstrated to be a negative and positive regulator, respectively, in the biosynthesis of ilamycin. Two overproducers were constructed by knockout of ilaA and overexpression of ilaB in S. atratus SCSIO ZH16. The secretion of ilamycins or self-defense of $S$. atratus SCSIO ZH16 to ilamycins by a pair of exporters encoded by ilaJ and ilaK was also characterized. Ilamycins were successfully produced in the Streptomyces model of S. coelicolor M1152. Our study on the regulatory and transport functions of IlaA/B/J/K not only sheds light on new characteristics of regulation, but also provides new genetic elements for synthetic biology.

\section{Material and Methods}

\subsection{Bacterial Strains, Plasmids, and Culture Conditions}

Bacterial strains and plasmids used in this study are listed in Table S1. E. coli strains, including DH5 $\alpha$ and ET12567/pUZ8002, were cultivated at $37^{\circ} \mathrm{C}$ in Luria-Bertani (LB) liquid medium or on LB agar. BW25113/pIJ790 was cultivated at $30^{\circ} \mathrm{C}$ or $37^{\circ} \mathrm{C}$ in super optimal broth (SOB) liquid medium, as necessary. S. atratus SCSIO ZH16 and its genetically engineered mutant strains were cultivated at $30{ }^{\circ} \mathrm{C}$ in P2 solid medium for sporulation and genetic manipulation [16]. When necessary, the medium was supplemented with apramycin $30 \mu \mathrm{g} / \mathrm{mL}$, chloramphenicol $25 \mu \mathrm{g} / \mathrm{mL}$, kanamycin $50 \mu \mathrm{g} / \mathrm{mL}$, thiostrepton $12.5 \mu \mathrm{g} / \mathrm{mL}$, or ampicillin $100 \mu \mathrm{g} / \mathrm{mL}$. 


\subsection{General Genetic Manipulations and Reagents}

General genetic manipulation of Streptomyces was carried out according to standard protocols. PCR amplification was performed on an Eppendorf Mastercycler ${ }^{\circledR}$ EP gradient (Eppendorf, Germany) using high-fidelity Taq DNA polymerase purchased from TransGen Biotech Co. Ltd (Beijing, China). DNA fragments and PCR products were purified from agarose gels using a DNA Gel Extraction Kit (Omega). Primers were synthesized by Sangon Biotech Co. Ltd. Company (Shanghai, China). All DNA sequencing was performed by IGE Biotech Co. Ltd (Guangzhou, China). Restriction enzymes and T4 DNA ligase were purchased from New England Biolabs (Ipswich, MA, USA).

\subsection{Genomic Library Screening}

The genomic cosmid library of S. atratus SCSIO ZH16 constructed with SuperCos1 has been described in our previous report [17]. Three pairs of primers (Table S2) were designed and used to screen the genomic cosmid library by PCR.

\subsection{Construction of Genetic Mutants}

All the mutant strains in this study were generated by homologous recombination according to the standard method [49]. For construction of the target mutants, the apramycin resistance cassette flanked by homologous arms with the target genes was amplified from the pIJ773 plasmid with Primer Star Taq enzymes (Takara) according to the manufacturer's specifications. The fragments were purified from the agarose gel using a gel recycle kit according to the manufacturer's specifications. The fragments were then electro-transformed into BW25113/pIJ790/plasmids. The disrupted plasmids were transformed into E. coli ET12567/pUZ8002 for conjugation. Interspecies conjugation was performed between E. coli ET12567/pUZ8002/disrupted plasmids and S. atratus SCSIO ZH16 on ISP4 solid medium supplemented with $20 \mathrm{mM} \mathrm{MgSO}_{4}$. Double-crossover mutants were identified through diagnostic PCR with corresponding primers listed in Table S2.

\subsection{Complementation of the $\triangle i l a B$ Mutant}

For complementation of the ilaB mutant, the target gene ilaB was cloned into the vector pl646ATE under the control of PermE*, which was digested with NdeI and SpeI, as reported by our group [34]. The correction of the target ilaB gene was verified by sequencing. The correctness of newly constructed complementary plasmids was verified by double enzyme digest, and the correct plasmids were transformed into E. coli ET12567/pUZ8002. Conjugation of E. coli ET 12567/ pUZ8002/ pl646ATE/ilaB with $\triangle i l a B$ mutant was then carried out. The correctness of conjugants was verified on the bases of the thiostrepton resistance phenotype and their genotypes.

For the construction of ilaB overexpression strain, the complemented plasmid, pl646ATE/ilaB, was introduced into S. atratus SCSIO ZH16 by conjugation with the aforementioned method. The correctness of the conjugants was verified on the bases of the thiostrepton resistance phenotype and their genotypes.

\subsection{Heterologous Expression of the Biosynthetic Gene Cluster of Ilamycins}

For heterologous expression of ilamycins, another genomic library was constructed with the pESAC13A vector by Bio S\&T, Germany; this vector can adopt large fragments ca 100-120 kbp [34,37]. To identify colonies containing the whole gene cluster of ilamcycins, three pairs of primers were designed for screening the genetic library by PCR. The desired colonies were used for conjugation with S. coelicolor M1152 using the triparental conjugation methods described by Tao et al. [36]. Correctness of the conjugants was verified by PCR analysis.

\subsection{Fermentation, Extraction, and Quantitative Analysis}

For fermentation, the spores/mycelium of S. atratus SCSIO ZH16 and its genetically engineered mutant strains were inoculated into Am2ab liquid medium [17] and cultivated at $30^{\circ} \mathrm{C}$ by shaking 
at $200 \mathrm{rpm}$ for 7 days. All the genetically engineered mutants were extracted with two-fold volume of butanone. The extracts were evaporated in vacuo and re-dissolved in $1 \mathrm{~mL}$ methanol for HPLC analysis, as described previously [16]. HPLC conditions are as follows: solvent system (solvent A, $15 \%$ acetonitrile in water supplemented with $0.1 \%$ acetic acid; solvent $\mathrm{B}, 85 \%$ acetonitrile in water supplemented with $0.1 \%$ acetic acid); $20 \%$ B to $80 \%$ B (linear gradient, $0-20 \mathrm{~min}$ ), $80 \%$ B to $100 \%$ B (linear gradient, $20-21.5 \mathrm{~min}), 100 \% \mathrm{~B}(21.5-27.0 \mathrm{~min}), 100 \%$ to $0 \% \mathrm{~B}(27.0-27.1 \mathrm{~min}), 0 \% \mathrm{~B}(27.1-30.0 \mathrm{~min})$; flow rate was set as $1.0 \mathrm{~mL} / \mathrm{min}$. A reverse phase column (SB-C18, $5 \mu \mathrm{m}, 4.6 \times 150 \mathrm{~mm}$ ) was used for analysis.

Quantitative analysis of ilamycin production was performed using GraphPad Prism 6 software. Ilamcyin yields were calculated using a calibration curve.

Supplementary Materials: The following are available online at http://www.mdpi.com/1660-3397/18/4/216/s1, Table S1: Summary of strains and plasmids used in this study; Table S2: Summary of primers used in this study; Figures S1, S2 and S5-S7: PCR verification of the disrupted genes ilaA, ilaB, ilaJ, ilaK, and ilaJK in the S. atratus SCSIO ZH16; Figures S3 and S4: the sequence alignment of IlaJ and IlaK with their homologues with the marked conserved domains or transmembrane domains; Figure S8: The quantitative HPLC standard curve for ilamycin D Figure S9: PCR verification of correct conjugants containing PAC7A6, which contains the entire ila gene cluster.

Author Contributions: J.M. and J.J. designed the experiments and revised the manuscript. J.H. constructed the gene inactivation mutants and complementation mutants and prepared the draft manuscript, X.W. conducted the bioinformatics analysis and constructed the over-expression strains, Y.L. carried out the heterologous expression of ila BGC, Z.Y. performed the verification and the fermentation of the mutants with the S. atratus SCSIO ZH16 as a control. All authors have read and agreed to the published version of the manuscript.

Funding: This research was funded by the National Natural Science Foundation of China (31870046, U1706206, 20872152); Special Funds for Economic Development of Marine Economy of Guangdong Province (GMDE-2018C003); the Key Special project for Introduced Talents Team of Southern Marine Science and Engineering Guangdong Laboratory (Guangzhou) (GML2019ZD0406) and the National Natural Science Foundation of Guangdong Province (2018A0303130005).

Conflicts of Interest: The authors declare no conflict of interest.

\section{References}

1. Jensen, P.R.; Moore, B.S.; Fenicala, W. The marine actinomycete genus Salinispora: A model organism for secondary metabolite discovery. Nat. Prod. Rep. 2015, 32, 738-751. [CrossRef] [PubMed]

2. Blunt, J.W.; Carroll, A.R.; Copp, B.R.; Davis, R.A.; Keyzers, R.A.; Prinsep, M.R. Marine natural products. Nat. Prod. Rep. 2018, 35, 8-53. [CrossRef] [PubMed]

3. Amoutzias, G.D.; Chaliotis, A.; Mossialos, D. Discovery strategies of bioactive compounds synthesized by nonribosomal peptide synthetases and type-I polyketide synthases derived from marine microbiomes. Mar. Drugs 2016, 14, 80. [CrossRef]

4. Agrawal, S.; Acharya, D.; Adholeya, A.; Barrow, C.J.; Deshmukh, S.K. Nonribosomal peptides from marine microbes and their antimicrobial and anticancer potential. Front. Pharmacol. 2017, 8, 828. [CrossRef] [PubMed]

5. Smolewski, P.; Robak, T. The discovery and development of romidepsin for the treatment of T-cell lymphoma. Expert Opin. Drug Discov. 2017, 12, 859-873. [CrossRef] [PubMed]

6. Maruyama, D.; Tobinai, K.; Ogura, M.; Uchida, T.; Hatake, K.; Taniwaki, M.; Ando, K.; Tsukasaki, K.; Ishida, T.; Kobayashi, N.; et al. Romidepsin in Japanese patients with relapsed or refractory peripheral T-cell lymphoma: A phase I/II and pharmacokinetics study. Int. J. Hematol. 2017, 106, 655-665. [CrossRef]

7. Sun, W.J.; Huang, H.; He, B.; Hu, D.H.; Li, P.H.; Yu, Y.J.; Zhou, X.H.; Lv, Z.; Zhou, L.; Hu, T.Y.; et al. Romidepsin induces G2/M phase arrest via Erk/cdc25C/cdc2/cyclinB pathway and apoptosis induction through JNK/c-Jun/caspase3 pathway in hepatocellular carcinoma cells. Biochem. Pharmacol. 2017, 127, 90-100. [CrossRef]

8. Eliopoulos, G.M.; Willey, S.; Reiszner, E.; Spitzer, P.G.; Caputo, G.; Moellering, R.C., Jr. In vitro and in vivo activity of LY 146032, a new cyclic lipopeptide antibiotic. Antimicrob. Agents Chemother. 1986, 30, $532-535$. [CrossRef]

9. Wanger, A.R.; Murray, B.E. Activity of LY146032 against Enterococci with and without high-level aminoglycoside resistance, including two penicillinase-producing strains. Antimicrob. Agents Chemother. 1987, 31, 1779-1781. [CrossRef] 
10. Mccormick, M.H.; Mcguire, J.M.; Pittenger, G.E.; Pittenger, R.C.; Stark, W.M. Vancomycin, a new antibiotic. I. Chemical and biologic properties. Antibiot. Annu. 1955, 3, 606-611. [PubMed]

11. Mcguire, J.M.; Wolfe, R.N.; Ziegler, D.W. Vancomycin, a new antibiotic. II. In vitro antibacterial studies. Antibiot. Annu. 1955, 3, 612-618.

12. Griffith, R.S.; Peck, F.B., Jr. Vancomycin, a new antibiotic. III. Preliminary clinical and laboratory studies. Antibiot. Annu. 1955, 3, 619-622.

13. Dijkstra, J.A.; van der Laan, T.; Akkerman, O.W.; Bolhuis, M.S.; de Lange, W.C.M.; Kosterink, J.G.W.; van der Werf, T.S.; Alffenaar, J.W.C.; van Soolingen, D. In Vitro Susceptibility of Mycobacterium tuberculosis to Amikacin, Kanamycin, and Capreomycin. Antimicrob. Agents Chemother. 2018, 62, e01724-17. [CrossRef] [PubMed]

14. Takeda Chemical Industries, LtD. Ruformycin. U.S. Patent 923938, 7 February 1961.

15. Takita, T.; Ohi, K.; Okami, Y.; Maeda, K.; Umezawa, H. New antibiotics, ilamycins. J. Antibiot. Ser. A 1962, 15, $46-48$.

16. Karl, P. Process for the isolation of ruformycin factors. Patent WO 00/78798 A1, 16 June 2000.

17. Ma, J.; Huang, H.; Xie, Y.; Liu, Z.; Zhao, J.; Zhang, C.; Jia, Y.; Zhang, Y.; Zhang, H.; Zhang, T. Biosynthesis of ilamycins featuring unusual building blocks and engineered production of enhanced anti-tuberculosis agents. Nat. Commun. 2017, 8, 391. [CrossRef] [PubMed]

18. Alaniappan, K.; Venkatraghavan, V. Rufomycin derivatives useful as antibiotics. Patent WO 00/78797 A1, 15 June 2000.

19. Choules, M.P.; Wolf, N.M.; Lee, H.; Anderson, J.R.; Grzelak, E.M.; Wang, Y.; Ma, R.; Gao, W.; McAlpine, J.B.; Jin, Y.Y.; et al. Rufomycin Targets ClpC1 proteolysis in Mycobacterium tuberculosis and M. abscessus. Antimicrob. Agents Chemother. 2019, 63, e02204-18. [CrossRef] [PubMed]

20. Xie, Q.; Yang, Z.; Huang, X.; Zhang, Z.; Li, J.; Ju, J.; Zhang, H.; Ma, J. Ilamycin C induces apoptosis and inhibits migration and invasion in triple-negative breast cancer by suppressing IL-6/STAT3 pathway. J. Hematol. Oncol. 2019, 12, 60. [CrossRef] [PubMed]

21. Zhou, W.; Fang, H.; Wu, Q.; Wang, X.; Liu, R.; Li, F.; Xiao, J.; Yuan, L.; Zhou, Z.; Ma, J.; et al. A natural product of marine actinomycete, inhibits triple-negative breast cancer partially through ER stress-CHOP-Bcl-2. Int. J. Biol. Sci. 2019, 15, 1723-1732. [CrossRef]

22. Tomita, H.; Katsuyama, Y.; Minami, H.; Ohnishi, Y. Identification and characterization of a bacterial cytochrome P450 monooxygenase catalyzing the 3-nitration of tyrosine in rufomycin biosynthesis. J. Biol. Chem. 2017, 292, 15859-15869. [CrossRef]

23. Huang, S.; Tong, M.H.; Qin, Z.; Deng, Z.; Deng, H.; Yu, Y. Identification and characterization of the biosynthetic gene cluster of thiolutin, a tumor angiogenesis inhibitor, in Saccharothrix algeriensis NRRL B-24137. Anticancer Agents Med. Chem. 2015, 15, 277-284. [CrossRef]

24. Marshall, C.G.; Broadhead, G.; Leskiw, B.K.; Wright, G.D. D-Ala-D-Ala ligases from glycopeptide antibiotic-producing organisms are highly homologous to the enterococcal vancomycin-resistance ligases VanA and VanB. Proc. Natl. Acad. Sci. USA 1997, 94, 6480-6483. [CrossRef]

25. Retzlaff, L.; Distler, J. The regulator of streptomycin gene expression, StrR, of Streptomyces griseus is a DNA binding activator protein with multiple recognition sites. Mol. Microbiol. 1995, 18, 151-162. [CrossRef] [PubMed]

26. Pootoolal, J.; Thomas, M.G.; Marshall, C.G.; Neu, J.M.; Hubbard, B.K.; Walsh, C.T.; Wright, G.D. Assembling the glycopeptide antibiotic scaffold: The biosynthesis of A47934 from Streptomyces toyocaensis NRRL15009. Proc. Natl. Acad. Sci. USA 2002, 99, 8962-8967. [CrossRef] [PubMed]

27. Madeira, F.; Park, Y.M.; Lee, J.; Buso, N.; Gur, T.; Madhusoodanan, N.; Basutkar, P.; Tivey, A.R.N.; Potter, S.C.; Finn, R.D.; et al. The EMBL-EBI search and sequence analysis tools APIs in 2019. Nucleic Acids Res. 2019, 47, W636-W641. [CrossRef] [PubMed]

28. Robert, X.; Gouet, P. Deciphering key features in protein structures with the new ENDscript server. Nucleic Acids Res. 2014, 42, W320-W324. [CrossRef]

29. El-Gebali, S.; Mistry, J.; Bateman, A.; Eddy, S.R.; Luciani, A.; Potter, S.C.; Qureshi, M.; Richardson, L.J.; Salazar, G.A.; Smart, A.; et al. The Pfam protein families database in 2019. Nucleic Acids Res. 2019. [CrossRef]

30. Menges, G.; Muth, W.; Wohelleben, W.; Stegmann, E. The ABC transporter Tba of Amycolatopsis balhimycina is required for efficient export of the glycopeptide antibiotic balhimycin. Appl. Microbiol. Biotechnol. 2007, 77, 125-134. [CrossRef] 
31. Kaur, P. Expression and characterization of DrrA and DrrB proteins of Streptomyces peucetius in Escherichia coli: DrrA is an ATP binding protein. J. Bacteriol. 1997, 179, 569-575. [CrossRef]

32. Fernandez, E.; Lombo, F.; Mendez, C.; Salas, J.A. An ABC transporter is essential for resistance to the antitumor agent mithramycin in the producer Streptomyces argillaceus. Mol. Gen. Genet. 1996, 251, 692-698.

33. Linton, K.J.; Cooper, H.N.; Hunter, I.S.; Leadlay, P.F. An ABC-transporter from Streptomyces longisporoflavus confers resistance to the polyetherionophore antibiotic tetronasin. Mol. Microbiol. 1994, 11, 777-785. [CrossRef]

34. Yang, Z.; Wei, X.; He, J.; Sun, C.; Ju, J.; Ma, J. Characterization of the noncanonical regulatory and transporter genes in atratumycin biosynthesis and production in a heterologous host. Mar. Drugs 2019, 17, 560. [CrossRef] [PubMed]

35. Kaur, P.; Rao, D.K.; Gandlur, S.W. Biochemical characterization of domains in the membrane subunit DrrB that interact with the ABC subunit DrrA: Identification of a conserved motif. Biochemistry 2005, 44, 2661-2670. [CrossRef] [PubMed]

36. Xu, M.; Wang, Y.; Zhao, Z.; Gao, G.; Huang, S.X.; Kang, Q.; He, X.; Lin, S.; Pang, X.; Deng, Z.; et al. Functional Genome mining for metabolites encoded by large gene clusters through heterologous expression of a whole-genome bacterial artificial chromosome library in Streptomyces spp. Appl. Environ. Microbiol. 2016, 82, 5795-5805. [CrossRef]

37. Tu, J.; Li, S.; Chen, J.; Song, Y.; Fu, S.; Ju, J.; Li, Q. Characterization and heterologous expression of the neoabyssomicin/abyssomicin biosynthetic gene cluster from Streptomyces koyangensis SCSIO 5802. Microb. Cell Fact. 2018, 17, 28. [CrossRef] [PubMed]

38. Newman, D.J.; Cragg, G.M. Natural products as sources of new drugs from 1981 to 2014. J. Nat. Prod. 2016, 79, 629-661. [CrossRef] [PubMed]

39. Cragg, G.M.; Newman, D.J. Natural products: A continuing source of novel drug leads. Biochim. Biophys. Acta 2013, 1830, 3670-3695. [CrossRef]

40. Bibb, M.J. Regulation of secondary metabolism in Streptomycetes. Curr. Opin. Microbiol. 2005, 8, $208-215$. [CrossRef]

41. Martín, J.F.; Liras, P. Engineering of regulatory cascades and networks controlling antibiotic biosynthesis in Streptomyces. Curr. Opin. Microbiol. 2010, 13, 263-273. [CrossRef]

42. Thamm, S.; Distler, J. Properties of C-terminal truncated derivatives of the activator, StrR, of the streptomycin biosynthesis in Streptomyces griseus. FEMS Microbiol. Lett. 1997, 149, 265-272. [CrossRef]

43. Tomono, A.; Tsai, Y.; Yamazaki, H.; Ohnishi, Y.; Horinouchi, S. Transcriptional control by A-factor of StrR, the pathway-specific transcriptional activator for streptomycin biosynthesis in Streptomyces griseus. J. Bacteriol. 2005, 187, 5595-5604. [CrossRef]

44. Lopez, D.; Vlamakis, H.; Kolter, R. Generation of multiple cell types in Bacillus subtilis. FEMS Microbiol. Rev. 2009, 33, 152-163. [CrossRef] [PubMed]

45. Davidson, A.L.; Dassa, E.; Orelle, C.; Chen, J. Structure, function, and evolution of bacterial ATP-binding cassette systems. Microbiol. Mol. Biol. Rev. 2008, 72, 317-364. [CrossRef] [PubMed]

46. Hollenstein, K.; Dawson, R.J.; Locher, K.P. Structure and mechanism of ABC transporter proteins. Curr. Opin. Struct. Biol. 2007, 17, 412-418. [CrossRef] [PubMed]

47. Zhu, Q.; Chen, Q.; Song, Y.; Huang, H.; Li, J.; Ma, J.; Li, Q.; Ju, J. Deciphering the sugar biosynthetic pathway and tailoring steps of nucleoside antibiotic A201A unveils a GDP-L-galactose mutase. Proc. Natl. Acad. Sci. USA 2017, 114, 4948-4953. [CrossRef]

48. Zhang, Y.; Huang, H.; Chen, Q.; Luo, M.; Sun, A.; Song, Y.; Ma, J.; Ju, J. Identification of the grincamycin gene cluster unveils divergent roles for GcnQ in different hosts, tailoring the L-rhodinose moiety. Org. Lett. 2013, 15, 3254-3257. [CrossRef] [PubMed]

49. Gust, B.; Challis, G.L.; Fowler, K.; Kieser, T.; Chater, K.F. PCR-targeted Streptomyces gene replacement identifies a protein domain needed for biosynthesis of the sesquiterpene soil odor geosmin. Proc. Natl. Acad. Sci. USA 2003, 100, 1541-1546. [CrossRef]

(C) 2020 by the authors. Licensee MDPI, Basel, Switzerland. This article is an open access article distributed under the terms and conditions of the Creative Commons Attribution (CC BY) license (http://creativecommons.org/licenses/by/4.0/). 